\title{
The impact of wealth status on dietary diversity of infants and young children in the Philippines
}

\author{
Yvonne M. Lenighan ${ }^{1}$, Imelda Angeles-Agdeppa ${ }^{2}$, Marvin B. Toledo ${ }^{2}$, Mario V. Capanzana ${ }^{2}$ \\ and Emma F Jacquier ${ }^{1}$ \\ ${ }^{1}$ Nestlé Research, Lausanne, Switzerland and \\ ${ }^{2}$ Food and Nutrition Research Institute, Manila, Philippines
}

\section{Abstract}

Introduction

Dietary diversity and socio-economic status are recognised as determinants of childhood malnutrition. The impact of wealth status on dietary diversity of infants and toddlers in Filipino infants and toddlers is currently unknown. Therefore, the aim of this analysis was to describe the impact of wealth status on dietary patterns using data from the 2013 Feeding Infants and Toddlers Study (FITS).

\section{Methods}

The FITS is a cross-sectional, national survey of infants $(6-11.9 \mathrm{mo} ; n=362)$, younger toddlers $(12-17.9 \mathrm{mo} ; n=360)$ and older toddlers $(18-23.9 \mathrm{mo} ; n=373)$. Dietary data were collected using 24-hour recalls. Percentage consumption of food groups were calculated. Data were stratified by socio-economic status (SES) based on the characteristics of their dwelling units.

\section{Results}

Fifty percent of the population were classified as low-SES, $20 \%$ middle-SES and $30 \%$ high-SES. Rice was the most commonly consumed food across all ages, followed by human milk and infant formula in infants and younger toddlers. Sixty-nine percent of infants from the low-SES consumed human milk compared to $41 \%$ of infants from the high-SES. In contrast, infant formula was consumed by $62 \%$ of high-SES infants compared to $25 \%$ of low-SES infants. Similarly, $54 \%$ of younger toddlers from the low-SES consumed human milk, compared to $24 \%$ of children from the high-SES, and only $10 \%$ of toddlers from poor-SES consumed toddler formula, compared to $47 \%$ of toddlers from the high-SES. Differences in non-milk based food groups were more evident in the older toddlers. Rice was consumed by approximately $90 \%$ of this population. Fish consumption varied by SES among toddlers (45\% in low-SES compared to $33 \%$ in high-SES). However, pork, chicken and sausages were consumed by less than $10 \%$ of toddlers from the low-SES compared to $18 \%, 16 \%$ and $25 \%$, respectively, from the high-SES. Vegetables were more highly consumed by toddlers from the low-SES (34\%) than the high-SES (27\%). Approximately $20 \%$ of older toddlers consumed cookies and $22 \%$ consumed sugar sweetened beverages, while cakes were consumed by $18 \%$ of toddlers from the high-SES compared to only $5 \%$ of toddlers from the low-SES.

\section{Discussion}

Dietary diversity in Filipino infants and toddlers is very limited. Rice, human milk and infant formula are the main source of nutrients for infants and young toddlers. Typically, there is low consumption of vegetables and protein-containing foods and high consumption of sugary foods, which is further impacted by SES. These data can be used to support public health initiatives to improve dietary diversity in the first years of life.

\section{Conflict of Interest}

YML and EFJ are Nestlé employees. 